\title{
Interrupted: The roles of distributed effort and incubation in preventing fixation and generating problem solutions
}

\author{
$\mathrm{Ut} \mathrm{Na} \mathrm{Sio}^{1} \cdot$ Kenneth Kotovsky $^{1} \cdot$ Jonathan Cagan $^{2}$
}

Published online: 27 December 2016

(C) Psychonomic Society, Inc. 2016

\begin{abstract}
Fixation on inappropriate concepts is a key barrier to problem solving. Previous research has shown that continuous work is likely to cause repeated retrieval of those concepts, resulting in increased fixation. Accordingly, distributing effort across problems through multiple, brief, and interlaced sessions (distributed effort) should prevent such fixation and in turn enhance problem solving. This study examined whether distributed effort can provide an advantage for problem solving, particularly for problems that can induce fixation (Experiment 1), and whether and how incubation can be combined with distributed effort to further enhance performance (Experiment 2). Remote Associates Test (RAT) problems were used as the problem-solving tasks. Half of them (i.e., misleading RAT) were more likely to mislead individuals to fixate on incorrect associates than the other half. Experiments revealed a superiority of distributed over massed effort on misleading RAT performance and a differing time course of incubation for the massed and distributed groups. We conclude that distributed effort facilitates problem solving, most likely via overcoming fixation. Cognitive mechanisms other than the commonly posited forgetting of inappropriate ideas may occur during incubation to facilitate problem solving.
\end{abstract}

Electronic supplementary material The online version of this article (doi:10.3758/s13421-016-0684-x) contains supplementary material, which is available to authorized users.

Ut Na Sio

unsio@cmu.edu

1 Department of Psychology, Carnegie Mellon University, Pittsburgh, PA 15213, USA

2 Department of Mechanical Engineering, Carnegie Mellon University, Pittsburgh 15213, PA, USA
The experiments in this article offer support for the occurrence of spreading activation during incubation.

Keywords Fixation · Problem solving · Distributed effort . Incubation

People constantly solve problems, from determining simple routes to work to solving societal problems, such as climate change. Although problems faced vary in terms of their scope and complexity, it is suggested that they can all be conceptualized as a process of search in a problem space (Newell \& Simon, 1972). When solving familiar problems, individuals can often efficiently use their relevant prior knowledge to generate a solution. However, for problems requiring nonroutine thinking, individuals often perform suboptimally (Davidson \& Sternberg, 2003). One factor that can account for the poor performance is individuals' tendency to fixate on irrelevant ideas (e.g., inappropriate prior knowledge or solutions for similar problems), with such fixation acting as a mental block preventing individuals from generating new solutions (Duncker, 1945; Smith \& Blankenship, 1991; Wiley, 1998).

\section{Problem solving and the persistence of fixation}

Solving problems often requires accessing information from memory relevant to the problems, including concepts that can be retrieved through automatic spreading activation within a semantic network or via more directed control processes (Dijksterhuis \& Nordgren, 2006; Hélie \& Sun, 2010). In semantic networks, concepts are interconnected according to associations among them (Anderson, 1983; Collins \& Loftus, 1975). During problem solving, activation originating 
from the problem will spread to their associated concepts. Close associates (e.g., our prior knowledge of similar problems) usually have strong and direct connections with the problem and thus receive a large amount of activation and are retrieved automatically. However, the automatic activation of these close associates may not always be useful. It often acts as a mental block preventing individuals from deliberately searching the semantic network to retrieve uncommon but useful ideas (Landau \& Lehr, 2004; Smith, 2003; Wiley, 1998). It should be noted that over time, activation may eventually spread through the semantic network to activate remote associates (Bowers, Regehr, Balthazard, \& Parker, 1990; Yaniv \& Meyer, 1987). However, during the initial problemsolving stage, close associates are likely to be activated first blocking the retrieval of more remote ideas.

Such fixation not only occurs naturally during problem solving; it is also likely to be accumulative. According to the activation-based memory model (Anderson, 1983), activation of an item increases each time it is retrieved and then slowly decays away. Recently retrieved concepts therefore have higher activation values than concepts that have not been retrieved. The high activation value will then make them more likely to be retrieved again. In other words, if individuals retrieve incorrect solutions initially, further work will often lead to repeated retrieval of the incorrect solutions, making it harder to think of new solutions. Such results have been shown in protocol studies (Cranford \& Moss, 2012; Moss, Kotovsky, \& Cagan, 2011).

\section{Distributed effort and overcoming fixation}

Given that working on one problem intensively for a considerable amount of time before moving on to another can increase fixation on inappropriate concepts, one potential way to prevent fixation build-up would be by solving problems in a distributed manner, where people break apart and distribute their effort across problems through multiple and short sessions interlaced with other problems.

Distributed practice is not a new technique. Numerous studies have shown that long-term memory is enhanced when learning events are spaced apart in time rather than massed in immediate succession; the effect is termed the "spacing effect" (Ebbinghaus, 1913; Janiszewski, Noel, \& Sawyer, 2003).

\section{Activation-based memory model of the spacing effect}

The spacing effect on memory retrieval has been explained on the basis of activation-based processes. According to the activation-based memory model, each time an item is practiced, it receives an increment in activation that then starts to decay. The rate of decay for each new practice is a function of the activation at the time of the presentation - high activation at the time of a practice trial will result in that practice trial decaying more quickly (Pavlik \& Anderson, 2005).

Massed-practice items will be highly activated because there is little time for the activation to decay between each practice. When practice trials are spaced, the activation of the item has time to decay between each practice. Distributed-practice items will therefore have a lower level of activation as compared to massed-practice items.

With regard to memory performance, although low activation may impair immediate recall of distributed-practice items, it is likely to enhance delayed recall. For the distributedpractice items, the increment for each added practice would decay slowly due to low activation level; the slow decay of the new practice should result in less forgetting over time and thus better long-term retention as compared to massed-practice items.

This study examined whether distributed effort can also facilitate other cognitive processing (i.e., problem solving). A distributed approach requires individuals to divide their effort across problems into multiple, brief, and interlaced sessions. If individuals fixate on incorrect concepts initially, switching to another problem might stop this potentially fruitless work, preventing the buildup of fixation. Also, according to the activation-based memory model, a distributed approach should provide time for the inappropriate concepts to decay between problem-solving attempts. This should enable individuals to start fresh and search in another direction when the problem is resumed, making the retrieval of remote concepts more likely. To test this prediction, Experiment 1 examined whether distributed practice can facilitate problem solving, particularly for problems that can induce fixation.

\section{Distributed effort and incubation}

In addition to the above effects on fixation, given the significant effects of spacing on delayed memory retrieval, distributed practice is likely to also affect delayed problem solving. There is increasing evidence (Baird, et al., 2012; Sio \& Ormerod, 2009; Zhong, Dijksterhuis, \& Galinsky, 2008) that incubation (Wallas, 1926), a period of time in which a problem is set aside before further attempts to solve, can facilitate subsequent problem solving. Different explanations have been advanced to explain such an effect.

One suggestion is that incubation provides time for information that obscures the solution to decay (the forgetting hypothesis; Smith, 1995; Smith \& Blankenship, 1991). Such forgetting should facilitate the retrieval of relevant ideas. Accordingly, a longer incubation interval should allow a greater degree of forgetting and thus yield a larger incubation effect. 
However, it is argued that relevant ideas are also likely to reduce in activation similarly to the irrelevant ones and thus be less available for retrieval (Ohlsson, 1992). Other mechanisms may occur during incubation to increase, or maintain, the activation level of relevant information.

One proposal is that the residual activation of the problem may spread through the semantic network during incubation to activate relevant remote associates (the spreading-activation hypothesis; Smith, 1995; Yaniv \& Meyer, 1987). If spreading activation is the mechanism underlying incubation, a longer incubation period would not necessarily generate a larger incubation effect. The optimal incubation interval needed for relevant remote associates to reach their peak level of activation would depend on the amount and decay rate of activation spreading from the problems to remote associates.

As proposed by the activation-based memory model, massed-practice items should be activated to a higher level, but decay more quickly. Similarly, problems presented in a massed fashion should possess a higher activation level and a faster decay rate. Therefore, for massed problems, a larger amount of activation should spread from the problems to the relevant remote concepts, but also diminish quickly during incubation, leading to a short-lived incubation effect. In contrast, spaced problems should have a lower level of activation, but decay more slowly. Therefore, the amount of activation spread from the problems to the remote concepts should be smaller but persist longer because of a slower decay rate. It should take more time for the remote concepts to be activated to a degree that can impact problem solving. Under this hypothesis, a long incubation interval would be needed for the distributed group, and a short one would be more helpful for the massed group.

In sum, if the effects of incubation are due to forgetting of inappropriate concepts, both the distributed and massed groups would benefit more from a longer incubation period. In contrast, according to the spreading-activation hypothesis, a short incubation period would be more helpful than a long one for the massed group. The forgetting and spreadingactivation hypotheses thus make different predictions of the optimal incubation interval for the massed group. Experiment 2 examined the effect of short and long incubation intervals for the massed and distributed groups. The results should provide evidence to test the forgetting and spreading-activation hypotheses.

\section{Overview of experiments}

Two experiments were conducted to examine the effects of distributed effort and incubation on problem solving. Experiment 1 investigated the basic effect of distributed effort on problem-solving performance. Experiment 2 examined the combined effects of distributed effort and incubation on problem-solving performance. Both distributed practice and incubation have been incorporated into various situations to promote learning and problem solving; yet they are often used separately rather than in combination (Carpenter, Cepeda, Rohrer, Kang, \& Pashler, 2012; Medd \& Houtz, 2002; Webster, Campbell, \& Jane, 2006). This study examining the question of whether and how the combination of them generates greater improvement thus should have direct implications for the development of methods to enhance productivity.

Remote Associate Test (RAT) problems (Mednick, 1962) were used as the problem-solving tasks in Experiments 1 and 2. The goal in RAT problems is to find a word that forms a compound word or common phrase with each of the three cues (e.g., cues: stick, maker, point; answer: match). The difficulty of the task lies in the fact that the cues and the answer are remotely rather than closely associated.

Recent studies (Moss et al., 2011; Smith, Huber, \& Vul, 2013) revealed that individuals solve RAT problems in a deliberate step-by-step manner. Individuals would first generate potential answers on the basis of one cue at a time, and then make new guesses based in part on their previous guesses. Words that are closely associated with the cues are likely to be generated first, and this may induce fixation that blocks the retrieval of remote answers. To retrieve the remote answers (e.g., via broadening the search or changing the search direction), individuals have to first disregard the incorrect close associates.

RAT problems can also be solved via unconscious processes, such as the spread of activation from the cues to the answer until the answer becomes activated to a degree that it can be retrieved. This often results in insightful solution discovery (Bowden \& Beeman, 2003a).

Experiment 1 examined the effect of distributed effort on initial problem solving. Experiment 2 examined whether and how incubation can be combined with distributed effort to further enhance performance. It is predicted that distributed effort impacts both initial and postincubation problem solving, though via different mechanisms. During initial problem solving, distributed effort should enhance RAT performance by reducing fixation and facilitating the deliberate search process. According to the activation-based memory model, massed and distributed problems should be activated to different levels after initial problem solving. If activation-based processes (i.e., spreading activation and forgetting) occur during incubation, a differing time course of incubation between the distributed and massed groups is expected.

\section{Experiment 1}

Experiment 1 examined whether distributed effort can enhance RAT problem solving, particularly for problems that can induce 
fixation on close associates. Two types (neutral vs. misleading) of RAT problems that differed in the types of cues given were used. The cues of misleading RAT problems were misleading in that they were more likely to induce fixation on common but incorrect associates, as compared to the cues of neutral RAT problems. If the benefit of distributed effort lies in preventing the accumulation of fixation on close associates, distributed effort should particularly facilitate the solving of misleading RAT problems. Also, the effect should become more visible over time as fixation builds in the massed group. We also analyzed separately how distributed effort impacts the insightful/unconscious and the noninsight/ deliberate problem-solving experience. If the benefit of distributed effort is to prevent fixation and in turn facilitate the deliberate search process, distributed effort should have a stronger impact for deliberate solutions than for insightful solutions (which are presumably the result of unconscious problem-solving processing).

\section{Method}

Participants Forty-eight ( 24 female, 24 male, mean age = 19.73 years, $S D=1.38$ ) native English-speaking undergraduates at Carnegie Mellon University participated for course credit.

Materials Ten neutral and 10 misleading RATs were selected from a normed set (Bowden \& Beeman, 2003b). A misleading RAT problem was one for which there was, in addition to the correct answer, a common misleading associate to only two of the three cues as determined by the Florida Association Norms (Nelson, McEvoy, \& Schreiber, 1998). For the example stick, point, maker, the common misleading associate was pin, which was associated with stick and point. The coappearance of the two cues (e.g., stick and point) should mislead the participant to fixate on their common but incorrect associates (e.g., pin), making it difficult to discover the remotely associated correct answer that links to all three cues (match for the example stick, point, maker). Similar manipulations have been shown to successfully induce fixation (Wiley, 1998; Sio \& Rudowicz, 2007).

The cues of misleading RAT problems were also linked to a larger number of close associates $(M=50.30, S D=6.67)$, as compared to the cues of neutral RAT problems $(M=42.30, S D$ $=9.93), p=.05$. This suggests that when solving misleading RAT problems, a greater number of incorrect associates is likely to be retrieved. To verify whether the manipulation worked at producing the desired result, a different set of 17 native English-speaking Carnegie Mellon University students (14 male, three female) with mean age 19.65 years $(S D=1.22)$ were run in a verbal protocol study in which they had to solve the RAT problems ( $30 \mathrm{~s}$ each) while simultaneously thinking aloud. The verbal protocols were coded for the frequency of the retrieval of common associates. As predicted, participants retrieved a higher proportion of close but incorrect associates when solving misleading $(M=.45, S D=.17)$ than neutral RAT $(M=.31, S D=.10)$ problems, $p=.006, d=1.00$. See Supplemental Material A for the misleading and neutral RAT problems used in this study.

Additional analyses were conducted to confirm that misleading and neutral RAT problems did not differ in other critical aspects. First, the strength of association between the cues and the answer- a measure of how closely the cues are to the answer-was assessed. No significance between misleading and neutral RAT problems was found, $p=.14$. Second, the associate frequency of the answers, which is an indicator of the prominence of words in memory (Griffiths, Steyvers, \& Firl, 2007), was examined. No significant difference was found between misleading and neutral RAT problems, $p=.93$.

Distributed versus massed effort Participants were randomly assigned to one of the two groups: massed versus distributed effort. For the massed group, each RAT problem was presented once for $30 \mathrm{~s}$. For the distributed group, RAT problems were presented repeatedly in three blocks. In each block, each RAT problem was presented once for $10 \mathrm{~s}$, intermixing with other RAT problems, and the correctly solved problems were removed in the next block. The maximum presentation time for each RAT problem was thus a total of $30 \mathrm{~s}$ in both groups.

Solution type Participants were instructed to judge whether they solved the RAT problem via an insightful or a noninsightful/deliberate search process after each correct solution. The instructions (see Supplemental Material B) for classifying a problem's solution as insightful or deliberate were adapted from Bowden and Beeman (2003a). Although this is a subjective measure to determine the type of processing that led to solution discovery, it has shown consistent differences in behavior and neural activity (Beeman \& Bowden, 2000; Kounios et al., 2008; Kounios et al., 2006).

Procedures Participants were randomly assigned to the massed $(30 \mathrm{~s} \times 1)$ or distributed $(10 \mathrm{~s} \times 3)$ group. Prior to the experiment, participants were instructed on the RAT problems and on the classification of solution type (insightful vs. deliberate), and were given four practice RAT problems to solve.

During each problem, the three cues were presented on a computer screen. Participants could enter their answers at any point. If their response was correct, they were prompted to judge whether they solved it with or without insight, and then the next problem was presented; otherwise, they had the remainder of the time limit to continue working on the problem.

\section{Results}

To examine the effect of distributed presentation on RAT performance, a mixed ANOVA was conducted on the number of 
correct responses on RAT problems using problem type (neutral or misleading) and solution type (deliberate or insightful) as the within-subjects factors, and presentation $(10 \mathrm{~s} \times 3$ or $30 \mathrm{~s}$ $\times 1)$ as the between-subjects factor.

Misleading versus neutral RAT The main effect of presentation was not significant, $F(1,46)=2.16, p=.15, \eta_{\mathrm{p}}{ }^{2}=.045$. Critically, there was a significant interaction between problem type and presentation, $F(1,46)=4.734, p=.035, \eta_{\mathrm{p}}{ }^{2}=.93$. The interaction was due to the significantly better performance on misleading RAT by the $10 \mathrm{~s} \times 3$ group $(M=4.22$, $S D=1.54)$, as compared with the $30 \mathrm{~s} \times 1$ group $(M=3.12, S D$ $=1.73), p=.03, d=.69$. Performance on neutral RAT problems was not significantly different between the $30 \mathrm{~s} \times 1(M=$ $3.48, S D=1.66)$ and $10 \mathrm{~s} \times 3(M=3.61, S D=1.60)$ groups, $p$ $=.79$. The interaction demonstrates that the facilitative effect of distributed effort was, as expected, observed only for misleading RAT problems (see Fig. 1).

Deliberate versus insight solution type If the benefit of distributed effort lies in reducing fixation on inappropriate associates and facilitating the deliberate search process, distributed effort should mainly enhance the number of problems solved via a noninsight/deliberate search process. Consistent with our expectation, there was a significant interaction between solution type and presentation, $F(1,46)=5.51, p=.023, \eta_{\mathrm{p}}{ }^{2}=$ .107. Follow-up ANOVAs revealed that the $10 \mathrm{~s} \times 3$ group $(M$ $=3.30, S D=2.24$ ) solved more RAT problems without insight than the $30 \mathrm{~s} \times 1$ group $(M=1.56, S D=1.50), F(1,46)=$ $10.16, p=.003, \eta_{\mathrm{p}}{ }^{2}=.108$. No significant distributed versus massed difference was found for the number of problems solved via insight, $F(1,46)=.98, p=.33, \eta_{\mathrm{p}}{ }^{2}=.021$. The interaction between problem type, solution type, and presentation was not significant, $p=.80$, implying similar patterns of results was observed across the problem types (see Fig. 2).

The benefit of distributed effort over time If it is the case that distributed effort can facilitate problem solving by preventing the buildup of fixation, the distributed versus massed performance difference for misleading RAT problems should increase over time as fixation builds in the massed

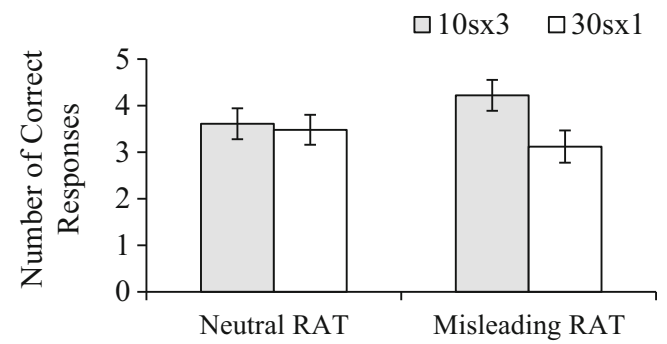

Fig. 1 RAT performance by presentation condition and problem type. Ten neutral and 10 misleading RAT problems were presented. Error bars indicate $+/-1$ standard error group. Figure 3 a show the cumulative proportion of misleading RAT problems solved during the $30 \mathrm{~s}$ in the $10 \mathrm{~s} \times 3$ and $30 \mathrm{~s} \times 1$ groups. Survival (Cox \& Oakes, 1984) and correlation analyses were conducted to examine if there was a significant and increasing difference between the $10 \mathrm{~s} \times 3$ and $30 \mathrm{~s} \times 1$ curves. A Kaplan-Meier survival analysis with a Mantel-Cox log-rank test confirmed a significant difference between these two curves, $p=.03$ (see Fig. 3a). The correlation analysis between the item exposure time and the size of the $10 \mathrm{~s} \times 3$ vs. $30 \mathrm{~s} \times 1$ difference revealed that the $10 \mathrm{~s} \times 3$ versus $30 \mathrm{~s} \times 1$ difference increased with time, $r(30)=.94, p<.001$.

For neutral RAT problems, the curves representing the cumulative proportion of RAT problems solved in the $10 \mathrm{~s} \times 3$ and $30 \mathrm{~s} \times 1$ groups largely overlapped (see Fig. $3 \mathrm{~b}$ ), $p=.85$ in the Kaplan-Meier analysis.

The differential effect of distributed effort for misleading and neutral RAT problems, together with our results on insightful vs. deliberate solution type comparisons and survival analysis, converge on our predictions that fixation on incorrect concepts gets stronger over time during massed problem solving and that a distributed approach helps prevent the build-up of such fixation, facilitating the deliberate search process. It is important to note that fixation is just one of the multiple causes of difficulty in problem solving (Kershaw \& Ohlsson, 2004). It is likely that different problem-solving strategies are needed for solving misleading and neutral RAT problems. The comparison of the performance of the massed and distributed groups on misleading RAT problems captures the facilitatory role of distributed effort in particular in overcoming fixation.

\section{Experiment 2}

In addition to replicating the benefit of distributed effort on RAT performance as revealed in Experiment 1, Experiment 2 was conducted to examine the combined effect of distributed effort and incubation on problem solving as well as the mechanisms underlying incubation. The effects of short (5-min) and long (10-min) incubation intervals were compared to examine the forgetting and spreading-activation hypotheses of incubation and to identify the optimal period of incubation for the massed and distributed groups. If forgetting of inappropriate concepts is the mechanism underlying incubation, a longer incubation period should generate greater postincubation improvement by providing more time for forgetting. According to the spreading-activation hypothesis, a longer incubation interval does not always yield a greater postincubation improvement. A massed group should benefit more from a short incubation interval while a distributed group should benefit more from a long one, due to the differing amount and decay rate of the activation of problems between the massed and distributed conditions. 


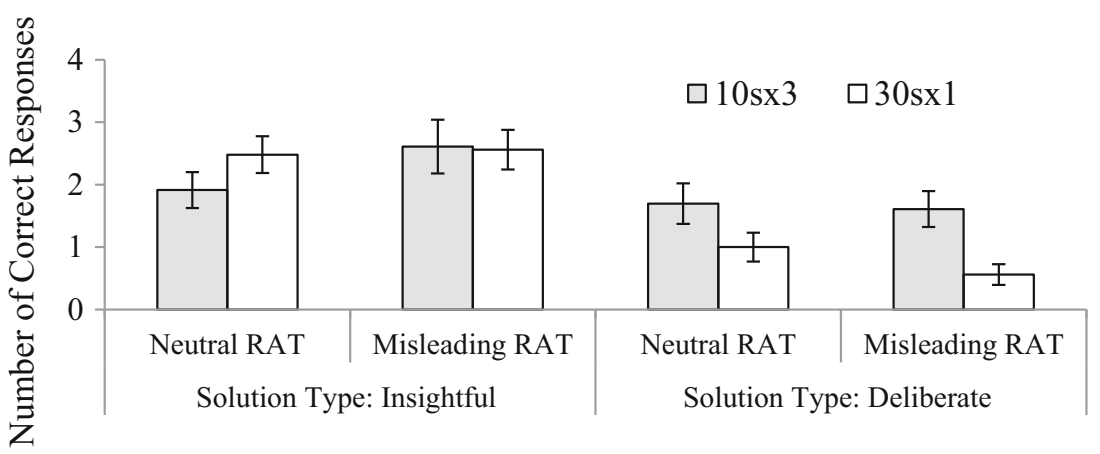

Fig. 2 RAT performance by presentation condition, solution type (self-judged), and problem type. Twenty RAT problems (10 neutral and 10 misleading) were presented. Error bars indicate $+/-1$ standard error

In addition to assessing RAT performance, we also adapted a lexical decision task (LDT; Meyer \& Schvaneveldt, 1971) to examine the spreading-activation hypothesis. In a LDT, individuals have to classify an item as a word or nonword as quickly as possible. It is suggested that individuals' lexical decision time reflects their sensitivity to that word. If activation would spread to activate relevant memory items during incubation, participants in the incubation conditions should make quicker lexical decisions to answers for RAT problems than those in the no-incubation condition.

\section{Method}

Participants One hundred and seventy-two (87 female, 85 male, mean age $=19.41$ years, $S D=1.56$ ) native Englishspeaking undergraduates at Carnegie Mellon University

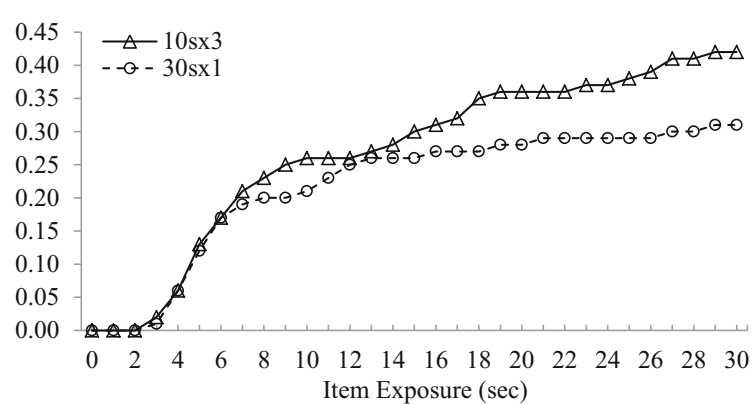

b

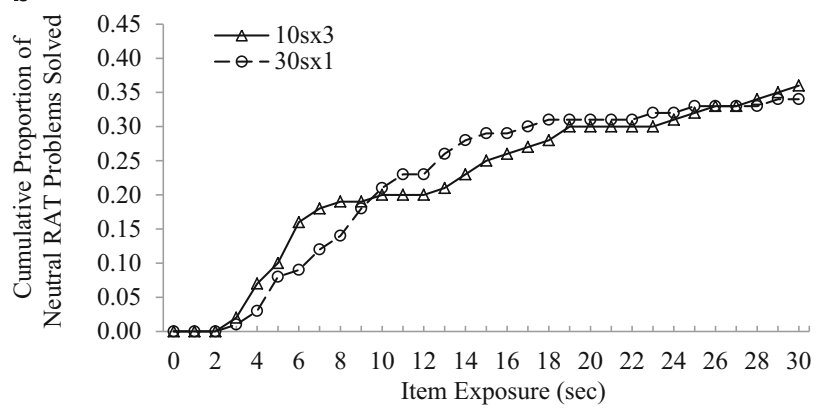

Fig. 3 a Cumulative proportion of misleading RAT problems solved by presentation condition. b Cumulative proportion of neutral RAT problems solved by presentation condition participated for course credit. Participants were randomly assigned to one of six conditions, differing in the length of incubation period (no-incubation, 5 minutes, or 10 minutes), and the presentation condition of RAT problems $(10 \mathrm{~s} \times 3$ or $30 \mathrm{~s} \times 1)$.

The average incubation effect size (Cohen's $d$ ) of the past incubation studies presenting RAT problems as the problemsolving tasks was .43 (Sio \& Ormerod, 2009). Having about 50 participants in each incubation condition would give us a power just over the threshold of $70 \%$ within the recommended range of power levels $(70 \%-80 \%)$ for finding incubation effects.

Materials and procedures The 20 RAT problems used in Experiment 1 and another 10 neutral RAT problems (see Supplemental Material A) selected from the normed set (Bowden \& Beeman, 2003b) were presented to participants in two sessions. As in Experiment 1, the cues of misleading RAT problems had a larger number of close associates $(M=$ $50.30, S D=6.67)$ than of neutral RAT problems $(M=39.30$, $S D=10.68), p=.006$, suggesting that more close but erroneous associates are likely to be retrieved when solving misleading RAT problems. No significant difference was found between misleading and neutral RAT problems in terms of the cue-answer associative strength, $p=.14$, and the associate frequency of the answers, $p=.78$.

In the first session, 10 misleading and 10 of the 20 neutral RAT problems were presented. In the second session, the unsolved and 10 remaining ("unseen") neutral RAT problems were presented. The number of "unsolved" RAT problems presented in the second sessions was different among participants, depending on the number of problems they solved during the first session. Therefore, the effect of incubation was assessed in terms of the proportion, rather than the number, of unsolved problems solved during the second session. Although the number of RAT problems presented in the second session varied at the individual level, it was similar across incubation conditions (see Table 1 in Supplemental Material C). Thus, this factor should not affect the general experimental manipulation. 
The neutral RAT problems assigned to the "unseen" set were counterbalanced between participants to ensure that each neutral RAT problem appeared equally often in both the unsolved and unseen cases. The procedures for presenting the RAT problems were the same as in Experiment 1.

The two sessions were separated either by intervening incubation tasks and a lexical decision task (5-min and 10-min incubation conditions), or a lexical decision task only (noincubation condition). Figure 4 presents the task sequence.

Visual search (Treisman \& Gelade, 1980) and mental rotation tasks (Shepard \& Metzler, 1971) were the incubation tasks in the incubation conditions. In each visual search task, participants had to judge if a target was present or absent in an image consisting of many objects. In each mental rotation task, participants had to judge if two images were identical or not. During the incubation period, individuals were first given practice visual search and mental rotation tasks. Then, visual search tasks were presented for 15 seconds each during the first 3 minutes of incubation, and mental rotation tasks were presented for 15 seconds each during the remaining incubation time. Participants could make their response at any point during the presentation. Presenting different types of tasks during incubation can make the incubation period less monotonous in order to help individuals stay focused. Both visual search and mental rotation tasks are spatial tasks that are very dissimilar to RAT problems, it is unlikely that they could produce a learning effect that could transfer to RAT problem solving. The amount of time spent on the visual search tasks was the same between the 5-min and 10-min incubation, and the only difference was the amount of time spent on the mental rotation tasks.

The lexical decision task (LDT; Meyer \& Schvaneveldt, 1971) was used to examine the spreading-activation hypothesis. If spreading activation is the source of incubation effects in RAT problem solving, enhanced performance should be accompanied by decreased lexical decision latencies to solution words. The LDT consisted of solution words (answers for half of the previously unsolved problems in each type of RAT problems), nonwords, and neutral words. The neutral words included 15 filler words unrelated to the RAT problems and solutions for half of the unseen RAT problems. The filler words were randomly selected from a set of 50 words that were of similar frequency and length as the answers to the RAT problems. Using similar items (answers of the RAT problems) as both solution and neutral words across participants minimizes bias due to uncontrolled differences between solution words and neutral words.

Enough nonwords were randomly selected from a pool of 160 nonwords so that there was an equivalent number of words and nonwords. The first 10 trials were warm-up trials that consisted of five filler words and five nonwords. The remaining trials consisted of neutral words, solution words, and nonwords presented in a randomized order. Each trial began with a fixation-cross presented in the center of the screen for $1,500 \mathrm{~ms}$ followed by the stimulus that remained on the screen until the response. A blank screen was presented for $500 \mathrm{~ms}$ between trials.

After the LDT, participants were prompted for the second session of RAT problems, which included the unsolved and unseen RAT problems. At the end, participants were asked if they noticed any relationship between the LDT and the RAT problems. Five participants in the distributed group (four in the no-incubation and one in the 5-min incubation condition) reported noticing the relationship between these two tasks. In the distributed group, all the unsolved problems were presented briefly before proceeding to the incubation tasks and the LDT. This may be the reason that the distributed group was more likely to notice the relationship between the LDT and the RAT problems. Because of noticing the relationship between these two tasks, they may incorporate the cue into problemsolving immediately once it was resumed, a process involving conscious awareness. This may confound the results of Experiment 2 examining the effect of incubation on problem solving. Data from these five participants were therefore excluded and analyzed separately.

\section{Results}

Distributed versus massed effort To test whether we could replicate the benefit of distributed effort on RAT performance

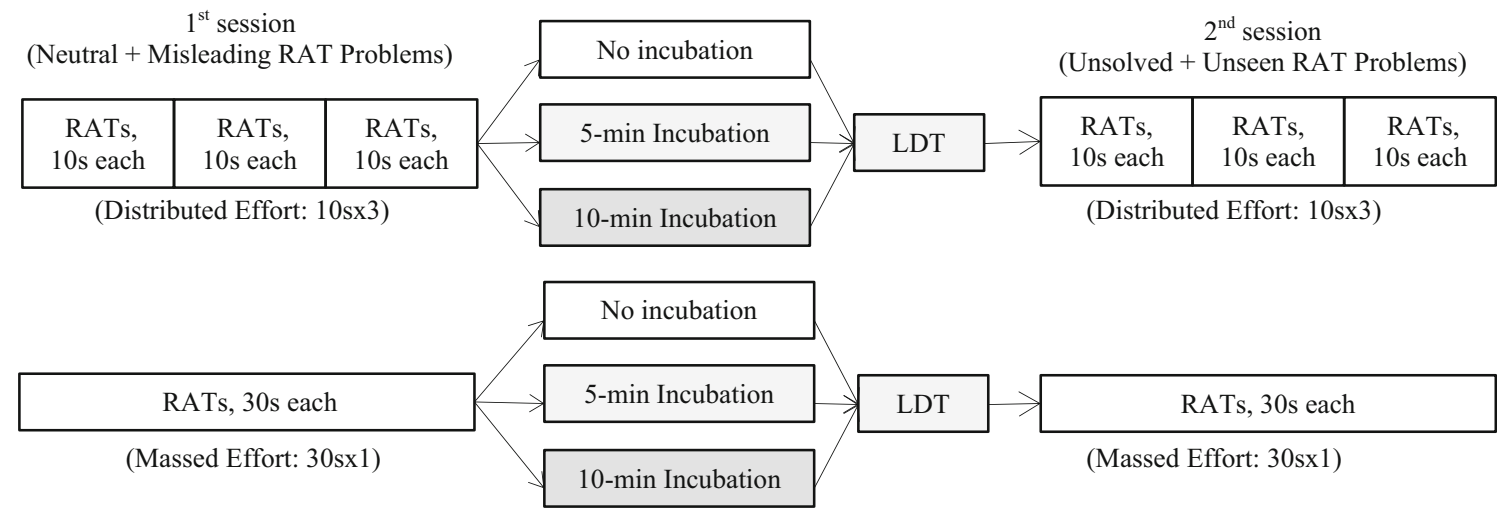

Fig. 4 Sequence of the tasks in the experiment 
as revealed in Experiment 1, a mixed ANOVA on the number of correct responses on RAT problems in the first session was conducted, with problem type (misleading or neutral) as a within-subjects factor, and presentation $(10 \mathrm{~s} \times 3$ or $30 \mathrm{~s} \times 1)$ and incubation (no incubation, 5-min incubation, or 10-min incubation) as between-subjects factors.

There was a significant problem type effect, $F(1,160)=$ $7.18, p=.008, \eta_{\mathrm{p}}^{2}=.043$, indicating that misleading RAT problems $(M=3.82, S D=1.89)$ were solved less often than neutral RAT problems $(M=4.20, S D=1.42)$. As expected, a significant interaction between problem type and presentation was found, $F(1,160)=6.97, p=.009, \eta_{\mathrm{p}}^{2}=.042$ (see Fig. 5). Follow-up ANOVAs revealed that the $10 \mathrm{~s} \times 3$ group $(M=$ $4.29, S D=1.91)$ solved more misleading RAT problems than the $30 \mathrm{~s} \times 1$ group $(M=3.35, S D=1.75), F(1,160)=10.67, p$ $=.001, d=.52, \eta_{\mathrm{p}}^{2}=.062$. No difference between the two groups for neutral RAT problems was found $(10 \mathrm{~s} \times 3: M=$ 4.30, $S D=1.54,30 \mathrm{~s} \times 1: M=4.11, S D=1.31), F(1,160)=$ $1.42, p=.40, \eta_{\mathrm{p}}^{2}=.004$. All other main and interaction effects were not significant, all $p>.20$, suggesting similar patterns of results was observed across the incubation conditions. This replication of Experiment 1 results would be expected as any differing manipulation from Experiment 1 took place after the first session.

The RAT performance in the first session for those (all in the $10 \mathrm{~s} \times 3$ condition) who reported noticing the relationship between the RAT problems and the lexical decision task were also examined. They solved $38 \%(S D=18.68)$ of neutral RAT problems and $42 \%(S D=14.16)$ of misleading RAT problems, which is comparable to the performance of the $10 \mathrm{~s} \times 3$ group in the analysis. Including these participants in the analyses did not change the direction or significance of the results.

Figure $6 \mathrm{a}$ and $\mathrm{b}$ show the cumulative proportion of misleading and neutral RAT problems solved during the $30 \mathrm{~s}$ in the first session in the $10 \mathrm{~s} \times 3$ and $30 \mathrm{~s} \times 1$ conditions. Again, the patterns resemble those reported in Experiment 1 that the 10s $\times 3$ vs. $30 \mathrm{~s} \times 1$ difference on misleading RAT problems increased as time elapsed (Fig. 6a), pointing to reducing levels of fixation as a likely explanation for the effect of distributed effort. A Kaplan-Meier analysis with a Mantel-Cox log-rank test revealed a significant difference between the $10 \mathrm{~s} \times 3$ and

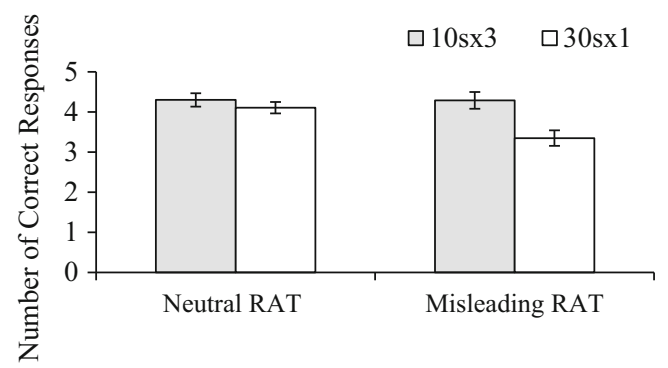

Fig. 5 RAT performance by presentation condition and problem type. Ten neutral and 10 misleading RAT problems were presented. Error bars indicate $+/-1$ standard error
$30 \mathrm{~s} \times 1$ curves for misleading RAT problems, $p=.001$, and such difference became larger as item exposure time increased, $r(30)=.92, p<.001$.

As in Experiment 1 , no significant $10 \mathrm{~s} \times 3$ vs. $30 \mathrm{~s} \times 1$ difference was observed for neutral RAT problems in the first session (Fig. 6b), $p=.50$ in the Kaplan-Meier analysis.

Distributed effort and time course of incubation The proportion of correct responses on previously unsolved RAT problem in the second session was analyzed in order to examine the incubation effect on problem-solving performance.

The factors presentation $(10 \mathrm{~s} \times 3$ or $30 \mathrm{~s} \times 1)$, incubation (no-incubation, 5-min incubation, or 10-min incubation), and problem type (neutral or misleading) were included in the analysis. To examine if the presentation of LDT containing answers for the RAT problems could confound the performance in the second session, the factor being whether or not the answer to the RAT problems (hint or no hint) had been presented in the lexical decision task was also included in the analysis. An ANOVA on the proportion of correct responses on previously unsolved RAT problems in the second session with all the above factors as independent variables was conducted.

The main effect of incubation was significant, $F(2,154)=$ 3.07, $p=.048, \eta_{\mathrm{p}}^{2}=.039$. A significant interaction between incubation and presentation was also found, $F(2,154)=4.04$, $p=.02, \eta_{\mathrm{p}}^{2}=.049$. Follow-up ANOVAs revealed a positive incubation effect for both the $30 \mathrm{~s} \times 1, F(1,76)=3.52, p=$ $.035, \eta_{\mathrm{p}}{ }^{2}=.085$, and10s $\times 3$ groups, $F(1,76)=3.52, p=.029$,

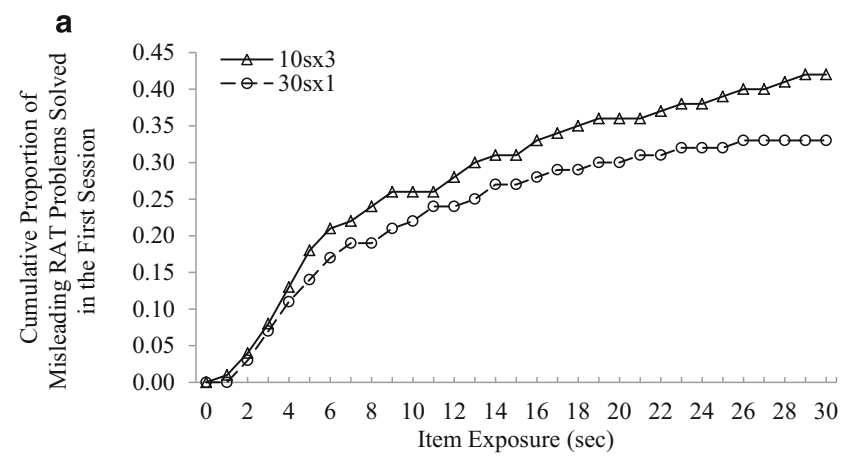

b

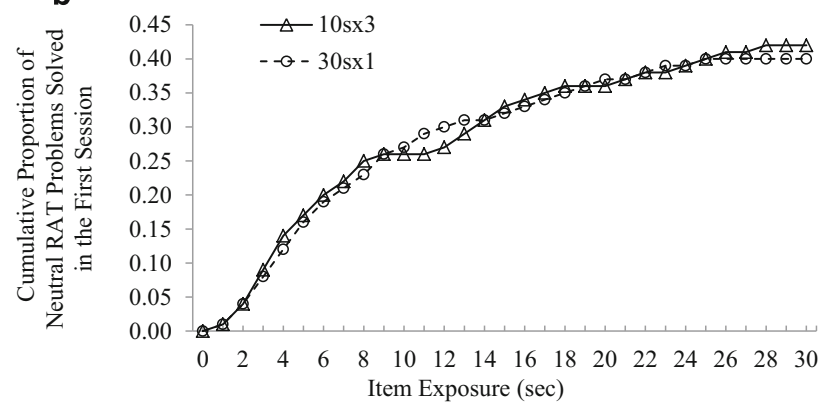

Fig. 6 a Cumulative proportion of misleading RAT problems solved by presentation condition $\mathbf{b}$ Cumulative proportion of neutral RAT problems solved by presentation condition 
$\eta_{\mathrm{p}}{ }^{2}=.087$. Tukey's post hoc comparisons revealed that these two groups exhibited a different optimal incubation time (see Fig. 7). For the $10 \mathrm{~s} \times 3$ group, the 10 -min incubation condition $(M=.27, S D=.14)$ performed significantly better than the noincubation condition $(M=.18, S D=.10), p=.04, d=.74$, and marginally better than the 5 -min incubation condition $(M=$ $.20, S D=.16), p=.12, d=.47$. No difference between the noincubation and 5-min incubation conditions was found, $p=$ 92. For the $30 \mathrm{~s} \times 1$ group, the 5-min incubation conditions ( $M$ $=.27, S D=.12$ ) performed better than the no-incubation condition $(M=.19, S D=.13), p=.03, d=.64$, and marginally better than the 10-min incubation condition $(M=.20, S D=$ $.13), p=.10, d=.56$; there was no difference between the 10 min incubation and no-incubation conditions, $p=.72$.

The differing time course of incubation for the $30 \mathrm{~s} \times 1$ and $10 \mathrm{~s} \times 3$ groups is in line with the prediction based on the spreading-activation hypothesis that the massed group would benefit most from a short incubation while the distributed group would benefit from a long one. This pattern of findings does not support the forgetting hypothesis predicting that a longer incubation interval should yield a greater incubation effect.

The effects of problem type and its interaction with other factors were not significant, all $p>.20$, implying that performance on misleading and neutral RAT problems were enhanced to the same degree after incubation.

Neither the main effect of hint nor its interactions with other factors were significant, all $p>.20$, indicating that whether or not the answer of the RAT problems was presented during the lexical decision task did not influence subsequent RAT performance.

To confirm that the incubation effect was specific to unsolved problems, we tested whether groups differed in terms of the number of unseen RAT problems solved. A 2 (problem type) $\times 2$ (hint) $\times 2$ (presentation) $\times 3$ (incubation) ANOVA revealed no significant differences (see Fig. 8), all $p s>.16$. This demonstrates that only unsolved problems benefited from incubation.

The five participants who noticed that relationship between the RAT problems and lexical decision task may have employed additional problem-solving strategies, such as recalling words presented in the lexical decision task while solving the problems. Their data were consistent with such a strategy because they improved more on misleading RAT problems where there was a hint (i.e., answer of RAT problems presented in the lexical decision task; proportion of correct responses with hint: $M=.38, S D=.19$, without hint: $M=$ $.15, S D=.22, p=.045$ ), and such difference was not found for those reported not noticing the relationship (with hint: $M=$ $.20, S D=.25$, without hint: $M=.21, S D=.27$ ).

In sum, both the $10 \mathrm{~s} \times 3$ and $30 \mathrm{~s} \times 1$ groups benefited from incubation (incubation effect size: $10 \mathrm{~s} \times 3: d=.74 ; 30 \mathrm{~s} \times 1: d$ $=.64)$ but in different timings $(30 \mathrm{~s} \times 1: 5-\mathrm{min}$ incubation, $10 \mathrm{~s}$ $\times$ 3: 10-min incubation). These findings are consistent with the general finding in the literature (Sio \& Ormerod, 2009) that incubation enhances problem solving. The findings of the
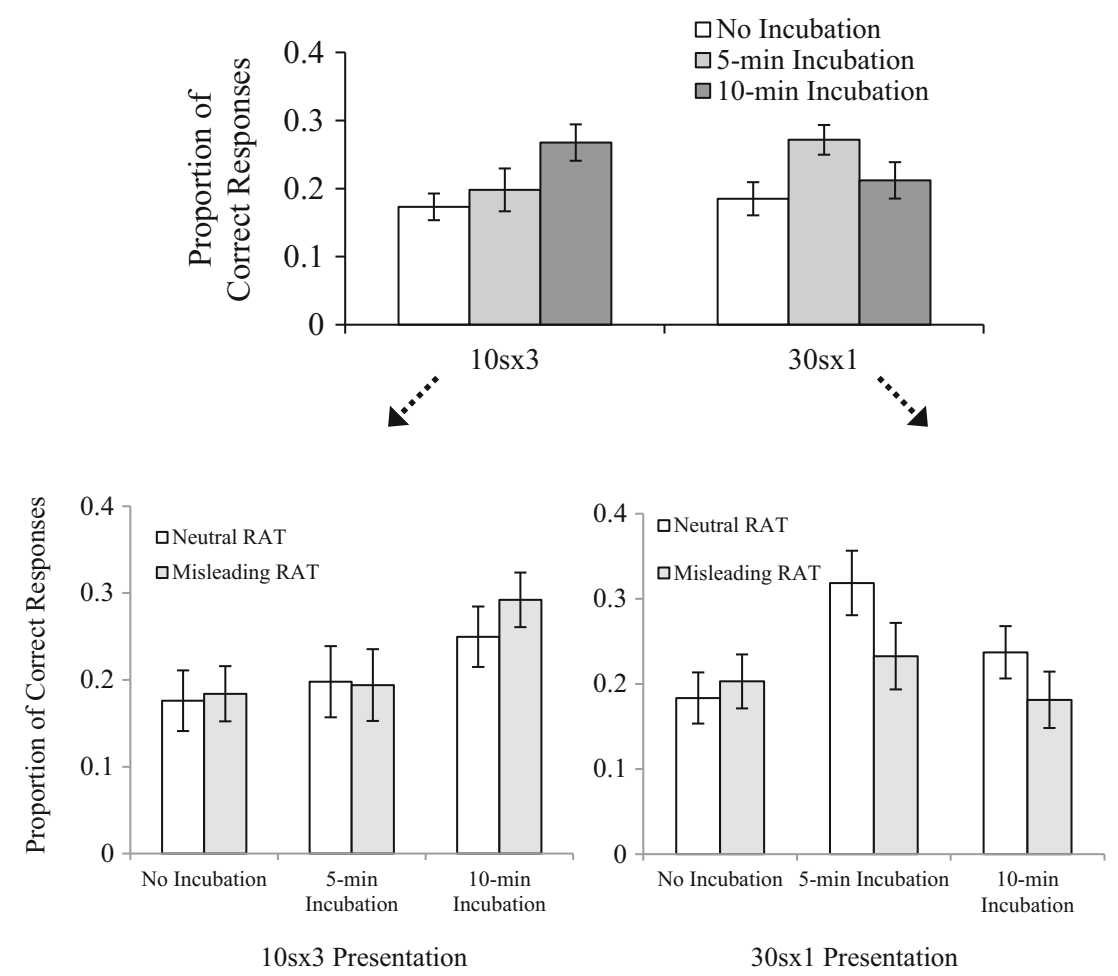

Fig. 7 Performance for unsolved RAT problems in the second session by presentation condition, incubation condition, and problem type. Error bars indicate $+/-1$ standard error 


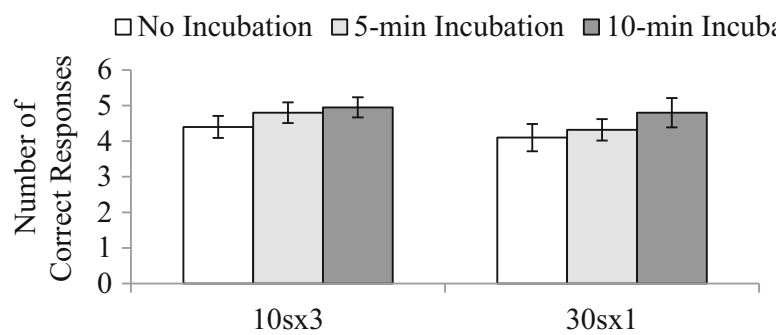

Fig. 8 Performance for unseen RAT problems (10 neutral RAT) in the second session by presentation condition and incubation condition. Error bars indicate $+/-1$ standard error

differential time course of incubation support the spreadingactivation hypothesis, which predicts that the incubation effect for the massed group will be short-lived and that the incubation effect for the distributed group will be slow to emerge.

Lexical priming To further test the spreading-activation hypothesis, participants' lexical decision times for RAT solution words were examined. If activation would spread in the semantic network to activate relevant memory items during incubation, the incubation groups should make quicker lexical decisions to RAT solution words than the no-incubation group.

Lexical decisions with extreme lexical decision time ( $<50 \mathrm{~ms}$ or $>1,300 \mathrm{~ms}$ ) were first discarded ( $1.6 \%$ of the data). Within each participant, any lexical decision time shorter or longer than 2 standard deviations from the mean was recoded to the value found at 2 standard deviations. The lexical decision times were then logarithmically transformed to further diminish skew. The transformed data were analyzed using a mixed ANCOVA with word type (the answer of unsolved misleading RAT or the answer to the unsolved neutral RAT) as a within-subjects factor, and presentation and incubation as between-subjects factors. To control for individual differences, log-transformed lexical decision times to neutral words (filler words and solutions of unseen RAT problems) were included as a covariate. None of the effects were significant, all $p \mathrm{~s}>.30$, indicating that participants in the incubation conditions did not make quicker lexical decisions to RAT solution words than those in the no-incubation conditions.

It may be the case that spreading activation occurs during incubation and facilitates problem solving only for some but not all unsolved RAT problems. Thus, a significant lexical priming effect may be found for items that were solved subsequently, but not on those that were unsolved. To examine this possibility, we conducted a one-way ANCOVA on the lexical decision times to answers of the previously unsolved RATs that were solved subsequently, with presentation and incubation as between-subjects factors, and log-transformed lexical decision times to neutral words as the covariate. No significant difference was revealed, all $p s>.25$.

In sum, the results of the first session performance replicated the findings of Experiment 1 that the $10 \mathrm{~s} \times 3$ groups solved more misleading RAT problems than the $30 \mathrm{~s} \times 1$ group. Both groups demonstrated a significant improvement on RAT performance after incubation, but with a different optimal incubation time. The findings on the post-incubation RAT performance favor the spreading-activation hypothesis over the forgetting hypothesis. However, the lexical decision data did not offer any additional evidence supporting the spreadingactivation hypothesis. We suggest that the absence of any lexical priming may be due to the insensitivity of the lexical decision task in detecting weak priming, which is discussed below.

\section{Discussion}

This study examined whether a distributed approach can facilitate problem solving (Experiment 1), and assessed the combined effects of distributed effort and incubation on problem solving (Experiment 2). Participants were asked to solve neutral and misleading RAT problems in the massed $(30 \mathrm{~s} \times 1)$ or distributed $(10 \mathrm{~s} \times 3)$ condition, with or without incubation. Both Experiment 1 and 2 revealed that the distributed group outperformed the massed one in solving misleading RAT problems. In addition to replicating the results of Experiment 1, Experiment 2 also showed that both massed and distributed groups benefited from incubation but in different timings (massed: 5-min incubation, distributed: 10-min incubation).

During problem solving, if individuals are fixated on inappropriate ideas initially, further work is likely to increase fixation. We suggest that approaching problems briefly and repetitively should avoid the build-up of that fixation and thus facilitate the retrieval of relevant but remote concepts. This proposition is supported by the findings that the distributed group outperformed the massed group in solving misleading RAT problems - problems that are likely to induce fixation, and that the advantage of distributed effort on misleading RAT problems became larger over time.

The effect of distributed practice on overcoming fixation can be explained on the basis of an activation-based memory model (Pavlik \& Anderson, 2005). When solving a problem, close associates are likely to be retrieved initially. Because concepts that have been retrieved recently have higher activation values than those that have not been retrieved, close associates are more likely to be retrieved repeatedly and lead to strong fixation. A distributed approach that disallows prolonged work on a problem could prevent such fixation. Also, it could provide time for these concepts to decay between problem-solving attempts, enabling individuals to start fresh again and search effectively to retrieve the remote concepts when the problem is resumed.

Forgetting can also be a consequence of interference (Altmann \& Gray, 2002). In addition to providing time for inappropriate concepts to decay between problem-solving 
attempts, the interleaved presentation of RAT problems may also facilitate interference-based forgetting. This does not contradict our proposition that distributed practice helps overcome fixation and instead suggests a possible additional role of distributed practice in overcoming fixation.

One may argue that the outperformance of the distributed $(10 \mathrm{~s} \times 3)$ group on misleading RAT problems might be attributed to other factors. In the distributed condition, RAT problems were presented repeatedly and interleaved with each other. With lots of words being activated across the RAT problems, the superior performance of the distributed group for misleading problems might thus be due to a largely activated semantic network rather than the overcoming of fixation. Accordingly, we should also expect an improvement on misleading RAT performance from the first to the second half of the massed condition in Experiment 1 and 2 (first session) as the semantic network should also be largely activated after solving half of the 20 RAT problems. However, such improvement was not observed in the massed condition: proportion of correct responses for misleading RAT: $M=.34, S D=.24$ (first half), $M=.32, S D=.32$ (second half), first versus second: $p=$ .33 ; neutral RAT: $M=.40, S D=.22$ (first half), $M=.41 S D=$ .39 (second half), first versus second: $p=.79$.

Another alternative explanation is that the $10 \mathrm{~s} \times 3$ presentation format might boost motivation at the beginning, leading to improved performance on subsequent problems. In the $10 \mathrm{~s}$ $\times 3$ condition, all the RAT problems were presented repeatedly in three blocks. It is likely that participants could solve some of the RAT problems during the first block. This might provide participants with a feeling of success, motivating them to perform better in the next block. The $30 \mathrm{~s} \times 1$ condition might not promote motivation to the same degree because only about one third (seven out of 20) of the RAT problems were presented to participants in the same period of time. However, note that the $10 \mathrm{~s} \times 3$ group also faced a higher number of failures than the $30 \times 1$ group during the first block. The $10 \mathrm{~s} \times 3$ group had about $75 \%$ (15 out of 20) RAT problems unsolved during the first block (see Figs. 6a and b). Given the high number of unsuccessful attempts, it is unlikely that the $10 \mathrm{~s} \times 3$ group would be more motivated after the first block.

Our results show that a distributed approach can facilitate problem solving. Having an incubation period can further enhance performance, as supported by the significant incubation effects observed in Experiment 2. The findings of Experiment 2 also advance our understanding of the role of incubation.

If the role of incubation is to allow inappropriate concepts to subside, a longer incubation interval should allow a greater decay of inappropriate concepts, resulting in a greater incubation effect. In our study, although the $10 \mathrm{~s} \times 3$ group did benefit more from a long incubation interval, the $30 \mathrm{~s} \times 1$ group benefited most from a short one. These findings do not support the forgetting hypothesis. The finding that the massed $(30 \mathrm{~s} \times 1)$ and distributed $(10 \mathrm{~s} \times 3)$ groups benefited most from 5-min and 10-min incubation, respectively, is more in line with the predictions on the basis of the spreading-activation hypothesis and the activation-based model of the spacing effect. According to the activation-based memory model, massed-practice items will be activated to a higher degree, but decay more quickly, as compared to distributed-practice items. Similarly, the cues of the RAT problems presented in the massed condition should be activated to a higher degree, but decay more quickly than in the distributed condition. Therefore, the answers in the $30 \mathrm{~s} \times 1$ condition should receive a large amount of activation from the cues initially during incubation, but the incoming activation should diminish quickly, resulting in an early but short-lived incubation effect. In contrast, the incoming activation for the answers in the $10 \mathrm{~s}$ $\times 3$ condition should be weaker, but decay more slowly. This explains the slower time course of incubation for the $10 \mathrm{~s} \times 3$ group as compared to the $30 \mathrm{~s} \times 1$ group. In sum, our findings suggest that spreading-activation occurs during incubation and that the time course of spreading-activation during incubation depends on the problem-solving approach (massed vs. distributed) individuals used during initial problem solving.

One may suggest that the inappropriate concepts associated with the problems may also decay more slowly in the $10 \mathrm{~s} \times 3$ group. The $10 \mathrm{~s} \times 3$ group benefited more from a long incubation period simply because this group needed a longer time for the inappropriate concepts to decay. According to this alternative, a longer incubation period should generate larger postincubation improvement by providing more time for forgetting. However, as was reported before, the $30 \mathrm{~s} \times 1$ group actually benefited most from a short incubation period. The spreading-activation account appears to be a better candidate to account for the differential time course of incubation.

One may doubt the spreading-activation account because Experiment 2 did not find any lexical decision priming. One possible reason for the null findings is that the lexical decision task is not very reliable in detecting weak priming. It has been suggested that lexical decision tasks are not simply a reflection of lexical access. Individuals may rely on other information (e.g., the meaningfulness of the words) to make the lexical decision (Balota \& Chumbley, 1985; Balota \& Lorch, 1986). This may decrease the sensitivity of the task for detecting weak priming (e.g., the spreading activation from the cues to the answers of the RAT problems). The ultimate resolution of this issue is likely to require more work using other sensitive and nondiscrimination measures (e.g., pronunciation task) in detecting weak priming.

Our findings also advance our understanding of the role of distributed practice in cognitive processing. A large body of research has shown that distributed presentation of information leads to better long-term learning and memory. Our findings reveal that a distributed approach can also facilitate problem solving. Studies of the spacing effect on memory and learning have identified numerous procedural factors (e.g., gaps between 
practices) that moderate the effectiveness of the distributed practice on memory recall. Further studies can examine if these factors also impact the effect of spacing on problem solving. For example, our study only included one distributed condition in which each RAT problem was presented for $10 \mathrm{~s}$ for 3 times, yet, it is likely that varying the presentation time and the gap between presentations may modify the size of the spacing effect on problem solving performance.

Distributed effort may also facilitate other problem-solving processes. Individuals tend to recall earlier problem solving experiences when they attempt a new problem, and recalling similar prior experiences may facilitate current problem solving due to some generalization formed from the comparison of the previous and current problems (Ross \& Kennedy, 1990; Ross, Perkins, \& Tenpenny, 1990). Distributing effort across problems through multiple and interlaced sessions may potentially facilitate generalization between problems. Also, recent studies have reported that interleaving presentation can enhance discrimination between items and thus enhance inductive learning (Birnbaum, Kornell, Bjork, \& Bjork, 2013; Kornell \& Bjork, 2008). Although generalization and discrimination are unlikely to happen in our study because there is no abstract schema underlying RAT problems, future studies could investigate whether distributed effort can facilitate abstract restructuring of problems affecting analogical transfer.

This study examined only accuracy and response time for RAT problems; future studies could collect more qualitative measures of problem solving to determine the nature of the effect of distributed effort on problem solving. For instance, previous studies have conducted protocol analysis on problem solving (Cranford \& Moss, 2012; Fleck \& Weisberg, 2004) and measured memory for unsolved problems (Monaghan et al., 2015; Patalano \& Seifert, 1994). These additional measures could indicate how distributed effort affects problem solving in greater detail, extending our current work that demonstrates the effect distributed effort has on problem solving.

Though RAT problems may best represent problem solving that involves specific linguistic representations, we suggest that our study provides valuable generalizations to many other problem-solving domains where disregarding irrelevant information is a critical feature for successful solution discovery (Gilhooly \& Murphy, 2005).

To conclude, our findings suggest that simply approaching problems briefly and repeatedly can facilitate problem solving, most likely via reducing fixation. Having an incubation period can further enhance performance. It is likely that problem-solving processes other than forgetting occur during incubation to enhance performance, and our study offers support, albeit not unequivocal, for that process being the occurrence of spreading activation during incubation.

Acknowledgements This research was supported by AFOSR Grant FA9550-16-1-0049 and FA9550-12-1-0374.

\section{References}

Anderson, J. R. (1983). A spreading activation theory of memory. Journal of Verbal Learning and Verbal Behavior, 22(3), 261-295.

Altmann, E. M., \& Gray, W. D. (2002). Forgetting to remember: The functional relationship of decay and interference. Psychological Science, 13(1), 27-33. doi:10.1111/1467-9280.00405

Baird, B., Smallwood, J., Mrazek, M. D., Kam, J. W. Y., Franklin, M. S., \& Schooler, J. W. (2012). Inspired by distraction: Mind wandering facilitates creative incubation. Psychological Science, 23(10), 1117 1122. doi:10.1177/0956797612446024

Balota, D. A., \& Chumbley, J. I. (1985). The locus of word-frequency effects in the pronunciation task: Lexical access and/or production? Journal of Memory and Language, 24(1), 89-106.

Balota, D. A., \& Lorch, R. F. (1986). Depth of automatic spreading activation: Mediated priming effects in pronunciation but not in lexical decision. Journal of Experimental Psychology: Learning, Memory, and Cognition, 12(3), 336-345.

Beeman, M. J., \& Bowden, E. M. (2000). The right hemisphere maintains solution-related activation for yet-to-be solved insight problems. Memory \& Cognition, 28(7), 1231-1241.

Birnbaum, M. S., Kornell, N., Bjork, E. L., \& Bjork, R. A. (2013). Why interleaving enhances inductive learning: The roles of discrimination and retrieval. Memory \& Cognition, 41(3), 392-402.

Bowden, E. M., \& Beeman, M. J. (2003a). Aha! Insight experience correlates with solution activation in the right hemisphere. Psychonomic Bulletin \& Review, 10(3), 730-737.

Bowden, E. M., \& Beeman, M. J. (2003b). Normative data for 144 compound remote associate problems. Behavior Research Methods, Instruments, \& Computers, 35(5), 634-639.

Bowers, K. S., Regehr, G., Balthazard, C. G., \& Parker, K. (1990). Intuition in the context of discovery. Cognitive Psychology, 22, 72-110.

Carpenter, S. K., Cepeda, N. J., Rohrer, D., Kang, S. H., \& Pashler, H. (2012). Using spacing to enhance diverse forms of learning: Review of recent research and implications for instruction. Educational Psychology Review, 24(3), 369-378.

Collins, A. M., \& Loftus, E. F. (1975). A spreading-activation theory of semantic processing. Psychological Review, 82(6), 407-428. doi:10.1037/0033-295x.82.6.407

Cox, D. R., \& Oakes, D. (1984). Analysis of survival data (Vol. 21). New York, NY: CRC Press.

Cranford, E. A., \& Moss, J. (2012). Is insight always the same? A protocol analysis of insight in compound remote associate problems. The Journal of Problem Solving, 4(2), 128-153. doi:10.7771/19326246.1129 .

Davidson, J. E., \& Sternberg, R. J. (Eds.). (2003). The psychology of problem solving. doi:10.1017/cbo9780511615771

Dijksterhuis, A., \& Nordgren, L. F. (2006). A theory of unconscious thought. Perspectives on Psychological Science, 1(2), 95-109. doi:10.1111/j.1745-6916.2006.00007.x

Duncker, K. (1945). On problem-solving. Psychological Monographs, 58(5), i-113. doi:10.1037/h0093599

Ebbinghaus, H. (1913). Memory. A contribution to experimental psychology. New York: Teachers College, Columbia University.

Fleck, J. I., \& Weisberg, R. W. (2004). The use of verbal protocols as data: An analysis of insight in the candle problem. Memory \& Cognition, 32(6), 990-1006. doi:10.3758/bf03196876

Gilhooly, K., \& Murphy, P. (2005). Differentiating insight from noninsight problems. Thinking \& Reasoning, 11(3), 279-302. doi:10.1080/13546780442000187

Griffiths, T. L., Steyvers, M., \& Firl, A. (2007). Google and the mind: Predicting fluency with PageRank. Psychological Science, 18(12), 1069-1076. 
Hélie, S., \& Sun, R. (2010). Incubation, insight, and creative problem solving: A unified theory and a connectionist model. Psychological Review, 117(3), 994-1024.

Janiszewski, C., Noel, H., \& Sawyer, A. G. (2003). A meta-analysis of the spacing effect in verbal learning: Implications for Research on advertising repetition and consumer memory. Journal of Consumer Research, 30(1), 138-149. doi:10.1086/374692

Kershaw, T. C., \& Ohlsson, S. (2004). Multiple causes of difficulty in insight: The case of the nine-dot problem. Journal of experimental psychology: Learning, memory, and cognition, 30(1), 3-13.

Kornell, N., \& Bjork, R. A. (2008). Learning concepts and categories: Is spacing the "enemy of induction"? Psychological Science, 19(6), 585-592. doi:10.1111/j.1467-9280.2008.02127.x

Kounios, J., Fleck, J. I., Green, D. L., Payne, L., Stevenson, J. L., Bowden, E. M., \& Jung Beeman, M. (2008). The origins of insight in resting-state brain activity. Neuropsychologia, 46(1), 281-291.

Kounios, J., Frymiare, J. L., Bowden, E. M., Fleck, J. I., Subramaniam, K., Parrish, T. B., \& Jung-Beeman, M. (2006). The prepared mind: Neural activity prior to problem presentation predicts solution by sudden insight. Psychological Science, 17, 882-890. Neuropsychologia, 46(1), 281-291. doi:10.1016/j. neuropsychologia.2007.07.013

Landau, J. D., \& Lehr, D. P. (2004). Conformity to experimenterprovided examples: Will people use an unusual feature? The Journal of Creative Behavior, 38(3), 180-191. doi:10.1002/j.21626057.2004.tb01239.x

Medd, E., \& Houtz, J. C. (2002). The effects of facilitated incubation on fourth graders' creative writing. Educational Research Quarterly, 26(2), 13-16.

Mednick, S. A. (1962). The associative basis of the creative process. Psychological Review, 69, 220-232.

Meyer, D. E., \& Schvaneveldt, R. W. (1971). Facilitation in recognizing pairs of words: Evidence of a dependence between retrieval operations. Journal of Experimental Psychology, 90(2), 227-234.

Monaghan, P., Sio, U. N., Lau, S. W., Woo, H. K., Linkenauger, S. A., \& Ormerod, T. C. (2015). Sleep promotes analogical transfer in problem solving. Cognition, 143, 25-30. doi:10.1016/j. cognition.2015.06.005

Moss, J., Kotovsky, K., \& Cagan, J. (2011). The effect of incidental hints when problems are suspended before, during, or after an impasse. Journal of Experimental Psychology: Learning, Memory, and Cognition, 37(1), 140-148.

Newell, A., \& Simon, H. A. (1972). Human problem solving. Englewood Cliffs, NJ: Prentice-Hall.

Nelson, D. L., McEvoy, C. L., \& Schreiber, T. A. (1998). The University of South Florida word association, rhyme, and word fragment norms. Retrieved from http://www.usf.edu/FreeAssociation/

Ohlsson, S. (1992). Information-processing explanations of insight and related phenomena. In M. T. Keane \& K. J. Gilhooly (Eds.),
Advances in the psychology of thinking (pp. 1-44). London, UK: Harvester Wheatsheaf.

Patalano, A. L., \& Seifert, C. M. (1994). Memory for impasses during problem solving. Memory \& Cognition, 22(2), 234-242. doi:10.3758/bf03208894

Pavlik, P. I., \& Anderson, J. R. (2005). Practice and forgetting effects on vocabulary memory: An activation-based model of the spacing effect. Cognitive Science, 29(4), 559-586.

Ross, B. H., \& Kennedy, P. T. (1990). Generalizing from the use of earlier examples in problem solving. Journal of Experimental Psychology: Learning, Memory, and Cognition, 16(1), 42-55.

Ross, B. H., Perkins, S. J., \& Tenpenny, P. L. (1990). Reminding-based category learning. Cognitive Psychology, 22(4), 460-492.

Shepard, R. N., \& Metzler, J. (1971). Mental rotation of threedimensional objects. Science, 171, 701-703.

Sio, U. N., \& Ormerod, T. C. (2009). Does incubation enhance problem solving? A meta-analytic review. Psychological Bulletin, 135(1), 94-120.

Sio, U. N., \& Rudowicz, E. (2007). The role of an incubation period in creative problem solving. Creativity Research Journal, 19(2/3), 307-318. doi:10.1080/10400410701397453

Smith, K. A., Huber, D. E., \& Vul, E. (2013). Multiply-constrained semantic search in the Remote Associates Test. Cognition, 128(1), 64 75.

Smith, S. M. (1995). Fixation, incubation, and insight in memory and creative thinking. In S. M. Smith, T. B. Ward, \& R. A. Finke (Eds.), The creative cognition approach (pp. 135-156). Cambridge, MA: MIT Press.

Smith, S. M. (2003). The constraining effects of initial ideas. In P. B. Paulus \& B. A. Nijstad (Eds.), Group creativity (pp. 15-31). New York, NY: Oxford University Press.

Smith, S. M., \& Blankenship, S. E. (1991). Incubation and the persistence of fixation in problem solving. American Journal of Psychology, 104, 61-87.

Treisman, A. M., \& Gelade, G. (1980). A feature-integration theory of attention. Cognitive Psychology, 12(1), 97-136.

Wallas, G. (1926). The art of thought. New York, NY: Harcourt, Brace.

Webster, A., Campbell, C., \& Jane, B. (2006). Enhancing the creative process for learning in primary technology education. International Journal of Technology \& Design Education, 16(3), 221-235.

Wiley, J. (1998). Expertise as mental set: The effects of domain knowledge in creative problem solving. Memory \& Cognition, 26, 716 730.

Yaniv, I., \& Meyer, D. E. (1987). Activation and metacognition of inaccessible stored information: Potential bases for incubation effects in problem solving. Journal of Experimental Psychology: Learning, Memory, and Cognition, 13, 187-205.

Zhong, C. B., Dijksterhuis, A., \& Galinsky, A. D. (2008). The merits of unconscious thought in creativity. Psychological Science, 19(9), 912-918. doi: 10.1111/j.1467-9280.2008.02176.x . 